\title{
Norovirus as the cause of medically attended gastroenteritis: a hospital-based experience
}

\author{
A. S. GASTAÑADUY ${ }^{1}$, J. ZABALETA ${ }^{1,2}$, L. LI $^{2}$ AND R. E. BÉGUÉ ${ }^{1 *}$ \\ ${ }^{1}$ Department of Pediatrics, Louisiana State University Health Sciences Center, New Orleans, LA, USA \\ ${ }^{2}$ Department of Genetics, and the Stanley S. Scott Cancer Center, Louisiana State University Health Sciences \\ Center, New Orleans, LA, USA
}

Received 31 August 2015; Final revision 18 January 2016; Accepted 28 February 2016;

first published online 16 March 2016

\section{SUMMARY}

Gastroenteritis remains an important cause of morbidity and mortality worldwide. With the introduction of vaccines against rotavirus, interest has shifted to understanding the epidemiology of norovirus (NoV). While the importance of NoV in gastroenteritis outbreaks is well established, its role in sporadic gastroenteritis is less known. To better define the role of NoV as a cause of sporadic gastroenteritis we investigated its prevalence in the patients seen in our paediatric hospital with special emphasis on its seasonal and age distribution. Over a 12-month period discarded stool specimens submitted to our paediatric hospital for testing of an infectious aetiology were retrieved and additionally tested for NoV by real-time reverse transcriptase-polymerase chain reaction; demographical and clinical information were also obtained. Overall, NoV was the single most commonly identified pathogen and found in 68/892 (7.6\%) total specimens or 68/258 (26\%) of pathogen-positive specimens. The highest rates of NoV were detected in children aged 6 months to 4 years $(50 / 332,15 \cdot 1 \%)$ and presenting between October and January $(46 / 314,14 \cdot 7 \%)$. NoV has become the main cause of gastroenteritis in our paediatric population.

Key words: Gastroenteritis, norovirus, paediatrics, surveillance.

\section{INTRODUCTION}

Despite ongoing progress, diarrhoea remains an important worldwide cause of mortality in children aged $<5$ years accounting for $9 \%$ or 0.6 million of the deaths [1]. Until recently rotavirus (RV) accounted for $\sim 50 \%$ of those diarrhoea-related deaths [2], but that is quickly changing now that RV vaccines are being introduced for childhood immunization. As expected, RV vaccines have been introduced more efficiently in developed countries and they are having

\footnotetext{
* Author for correspondence: R. E. Bégué, MD, Division of Infectious Diseases, Children's Hospital, 200 Henry Clay Avenue, New Orleans, LA 70118, USA.

(Email: rbegue@1suhsc.edu)
}

a significant impact in the amount of RV disease. For example at our hospital, the rate of hospitalizations due to gastroenteritis in general and to $\mathrm{RV}$-specific gastroenteritis decreased by $\sim 50 \%$ and the number of gastroenteritis episodes seen at the doctor's office decreased by $27 \%$, after only $55 \%$ utilization of the vaccine [3]. These findings have been extended and corroborated by national surveillance studies in the United States [4] and across European countries [5].

As RV diarrhoea decreases, the role of other viruses is becoming more evident. The second most common viral cause of diarrhoea is norovirus $(\mathrm{NoV})$. NoV is a small $(27 \mathrm{~nm})$ human calicivirus; the prototype virus, Norwalk virus, was identified in 1972 by electron microscopy [6]. Detection of NoV particles, and hence understanding of its epidemiology, was rather difficult 
until the early 2000s when virus-specific conventional reverse transcriptase-polymerase chain reaction (RTPCR) was developed. The introduction of RT-PCR soon identified NoV as the leading cause of epidemic (outbreak) gastroenteritis, affecting all age groups, and causing $>90 \%$ of non-bacterial and $\sim 50 \%$ of allcause epidemic gastroenteritis worldwide [7, 8].

But, while the role of NoV in epidemic diarrhoea was soon established, its role in endemic (sporadic) diarrhoea is less well characterized - especially in children. A literature review in 2008 [9] identified 31 studies (published between 1997 and 2008) that met quality inclusion criteria (duration for $>1$ year and use of RT-PCR), and found that NoV accounted for $12 \%(5-36 \%)$ of cases of mild and moderate diarrhoea and $11 \%$ (3-31\%) of severe diarrhoea. The studies corresponded to various countries and age groups. Only one study [10] was from the United States, which included children aged $<4$ years hospitalized for diarrhoea and found NoV in $7 \cdot 1 \%$ of the cases. A more recent similar systematic review in 2014 [11] identified 175 studies (published between 2008 and 2014) from 48 countries and estimated a pooled NoV prevalence of $18 \%(95 \%$ confidence interval $17-20 \%)$ for all cases of acute gastroenteritis. In the United States specifically, investigators from the Centers for Disease Control and Prevention (CDC) analysed an insurance claims database including various public and private health plans (Medicaid and uninsured populations not included) covering about 30 million persons in all 50 states and estimated that NoV caused $13 \%$ of all gastroenteritis-associated visits [12]. However, these are estimates performed using sophisticated statistical models and need to be corroborated with more conventional epidemiological studies.

The dearth of information on the role of $\mathrm{NoV}$ as cause of sporadic gastroenteritis in the United States prompted us to investigate its prevalence in patients seen in our paediatric hospital (outpatient and inpatient departments) with special emphasis on its seasonal and age distribution.

\section{METHODS}

\section{Study site}

Children's Hospital New Orleans, a 247-bed university-affiliated hospital, is the largest single paediatric facility in the state of Louisiana, admitting $54 \%$ of paediatric patients in the New Orleans area. Annually, $\sim 70000$ children receive care at our hospital and $\sim 1200$ stool specimens are submitted to the clinical laboratory for evaluation of a pathogen.

\section{Data collection}

The list of all stool samples submitted for microbiological analysis was reviewed three times a week by one of the authors (R.E.B.) and patient-identifying information was extracted. By regulation, the laboratory saves specimens for $72 \mathrm{~h}$ before being discarded. After all clinical tests had been completed and before discarding, the specimen was retrieved for further $\mathrm{NoV}$ analysis. Stool specimens were stored at $+4{ }^{\circ} \mathrm{C}$ in the laboratory until retrieved and then stored at $-70{ }^{\circ} \mathrm{C}$ until tested for NoV.

Using the identifiers, the clinical records corresponding to the gastroenteritis encounter were retrieved and information collected regarding duration and severity of diarrhoea, associated symptoms, need for hospitalization and laboratory results. Full information was available for children seen in the emergency department or hospitalized. For children seen in the outpatient clinics, full demographical information was available but only limited information on symptoms or clinical findings.

\section{NoV testing}

NoV testing was performed by real-time RT-PCR following a protocol previously described by Kageyama et al. [13] and adapted by the CDC (kindly provided by Dr Pengbo Liu, Emory University, Atlanta, GA). Briefly, stool specimens were thawed, a $10-20 \%$ suspension prepared in DNAse/RNase-free water, centrifuged and the supernatant used to purify RNA using QIAamp Viral RNA Mini kit (Qiagen, USA) and stored at $-70{ }^{\circ} \mathrm{C}$. The resulting viral RNA concentration was measured and $1 \mu \mathrm{g}$ subjected to reverse transcription (Superscript II Reverse Transcriptase; Invitrogen, USA) with random primers (Random Primers, Invitrogen). The resulting cDNA was included in the PCR reaction (Taqman Universal PCR Mastermix, Applied Biosystems, USA) and amplified in a thermocycler (QuantStudio 12Kplex, Applied Biosystems). Amplification reactions were run in duplicate and with positive and negative controls (provided by CDC). For NoV Genogroup I (GI), the primers COG1 F and COG1R and the probes RingG1(a) and RingG2(b) were used; for $\mathrm{NoV}$ GII, the primers COG2 F and COG2R and the probe RingG2-TP were used [13]. The PCR activation step used $95^{\circ} \mathrm{C}$ for $15 \mathrm{~min}$, followed by 
45 amplification cycles at $95^{\circ} \mathrm{C}$ for $15 \mathrm{~s}$ for denaturing and $56^{\circ} \mathrm{C}$ for $1 \mathrm{~min}$ for annealing/extension. Any cycle threshold signal indicated a possible positive result.

Specimens with a positive signal by real-time RT-PCR were also subjected to conventional RTPCR [14], the resulting amplicon run in an agarose gel (for confirmation of correct size), purified (QIAquick PCR Purification kit, Qiagen) and sequenced (Stanley S. Scott Cancer Center's Translational Genomic Core, LSUHSC, New Orleans, LA) for identification as NoV and subtyping by comparing the DNA sequence to databases at NIH's GenBank (http://www.ncbi.nlm.nih.gov/genbank) and the Norovirus Genotyping Tool (http:// www.rivm.nl/mpf/norovirus/typingtool).

\section{Data analysis}

In the event that multiple stool specimens were submitted for the same patient, only the first specimen was retrieved and tested. All specimens submitted within a 14-day period were considered as part of the same gastroenteritis episode (and only one tested). Cases were classified as community-acquired gastroenteritis if the specimen was obtained from an outpatient or within the first 3 days of admission, or hospital-acquired gastroenteritis if the specimen was collected $>72 \mathrm{~h}$ after admission and the patient had not been admitted for acute gastroenteritis. Specimens from cases of hospital-acquired gastroenteritis were collected and tested for NoV but are presented separately since they represent a different clinical entity.

The frequency and seasonality of NoV infection (monthly occurrence) was calculated and compared; as well as frequency for age subgroups. Tables depicting absolute and percent frequency of the clinical and epidemiological variables were constructed. Proportion variables were compared with $\chi^{2}$ with Yates' correction or Fisher's exact test, as appropriate (Statcalc, Epi-Info v. 3.5; CDC, USA); continuous variables were compared with the non-parametric Mann-Whitney $U$ test (Prism v. 5.04; GraphPad, USA).

\section{Ethical standards}

The study was reviewed and approved by the Institutional Review Board of Louisiana State University Health Sciences Center and Children's Hospital, both in New Orleans, LA, USA. The authors assert that all procedures contributing to this work comply with the ethical standards of the relevant national and institutional committees on human experimentation and with the Helsinki Declaration of 1975, as revised in 2008.

\section{RESULTS}

During the 12-month period (1 June 2011 to 31 May 2012), 1136 stool specimens were submitted to the hospital's laboratory that met the study inclusion criteria; and of these, $975(85.8 \%)$ specimens from 791 patients were available for testing.

Of the 975 specimens, 83 (8.5\%) (from 68 unique patients) were classified as hospital-acquired gastroenteritis. Sixty-five $(78.3 \%)$ specimens were tested for C. difficile toxin (ten found positive), $63(75.9 \%)$ were cultured for bacteria (one found positive for Shigella sonnei), $56(67 \cdot 5 \%)$ were tested for RV/adenovirus antigen (one found positive for adenovirus) and one was tested for GiardialCryptosporidium antigen (found negative). All 83 hospital-acquired gastroenteritis specimens were tested for $\mathrm{NoV}$ and none was positive.

The remaining 892 specimens represented community-acquired gastroenteritis, $552 \quad(61.9 \%)$ seen on an outpatient basis only (either clinics or emergency department and discharged) and 340 (38.1\%) requiring hospitalization. All 892 specimens were tested for NoV, while $748(83.9 \%), 534$ $(59 \cdot 9 \%), 312(35 \cdot 0 \%)$ and $163(18 \cdot 3 \%)$ were additionally tested for bacterial culture, RV/adenovirus antigen, $C$. difficile toxin or parasites, respectively. At least one pathogen was identified in $258(28.9 \%)$ specimens, corresponding to 240 distinct patients. NoV was detected in 68 cases, accounting for $7 \cdot 6 \%$ of all the specimens or $26.4 \%$ of the pathogen-positive ones. Numerically, NoV was the most commonly identified pathogen (68) followed by $C$. difficile (62), Salmonella spp. (46) and Shigella spp. (45); others were detected in much lower numbers (Table 1). RV was isolated from only $16(2.9 \%)$ of 534 specimens. Since testing was selective for pathogens other than NoV, the percent positivity varied by pathogen and was highest for $C$. difficile $(19 \cdot 9 \%)$ followed by $\mathrm{NoV}(7 \cdot 6 \%)$ (Table 1).

The most common NoV genogroup was GII, seen in $56(82 \cdot 3 \%)$ specimens followed by GI, seen in 11 $(16 \cdot 2 \%)$ specimens, with one $(1 \cdot 5 \%)$ specimen testing positive for both GI and GII. The most common genotype was GII.4 seen in $44(64 \cdot 7 \%)$ specimens. The 68 NoV-positive specimens corresponded to 
Table 1. Results of microbiological studies

\begin{tabular}{|c|c|c|c|c|}
\hline Group & Subgroup & $\begin{array}{l}\text { No. } \\
\text { tested }\end{array}$ & $\begin{array}{l}\text { No. } \\
\text { positive }\end{array}$ & $\begin{array}{l}\% \\
\text { positive }\end{array}$ \\
\hline \multirow[t]{8}{*}{ Bacteria } & $\begin{array}{l}\text { Clostridium difficile } \\
\text { toxin* }\end{array}$ & 312 & 62 & $19 \cdot 9$ \\
\hline & Salmonella spp.† & 748 & 46 & $6 \cdot 2$ \\
\hline & Shigella spp.† & 748 & 45 & $6 \cdot 0$ \\
\hline & $\begin{array}{l}\text { Campylobacter } \\
\text { spp. } \dagger\end{array}$ & 748 & 4 & $0 \cdot 53$ \\
\hline & $\begin{array}{l}\text { Escherichia coli } \\
\mathrm{O} 157 \dagger\end{array}$ & 748 & 1 & $0 \cdot 13$ \\
\hline & $\begin{array}{c}\text { Cronobacter } \\
\text { sakazakii† }\end{array}$ & 748 & 1 & $0 \cdot 13$ \\
\hline & Aeromonas caviae $\dagger$ & 748 & 1 & $0 \cdot 13$ \\
\hline & Edwardsiella tarda $\dagger$ & 748 & 1 & $0 \cdot 13$ \\
\hline \multirow[t]{3}{*}{ Viruses } & Norovirus: & 892 & 68 & $7 \cdot 6$ \\
\hline & $\begin{array}{l}\text { Adenovirus } \\
\text { antigen } \S\end{array}$ & 534 & 25 & $4 \cdot 7$ \\
\hline & Rotavirus antigen\| & 534 & 16 & $3 \cdot 0$ \\
\hline \multirow[t]{3}{*}{ Parasites } & $\begin{array}{l}\text { Cryptosporidium } \\
\text { antigen }\end{array}$ & 163 & 5 & $3 \cdot 1$ \\
\hline & Giardia antigen & 163 & 2 & $1 \cdot 2$ \\
\hline & $\begin{array}{l}\text { Blastocystis } \\
\text { hominis\# }\end{array}$ & 163 & 2 & $1 \cdot 2$ \\
\hline
\end{tabular}

* By nucleic acid amplification (Illumigene C. difficile; Meridian Bioscience Inc., USA).

$\uparrow$ By in-house culture.

+ By RT-qPCR (as described in Methods section).

$\S$ By enzyme immunoassay (Premier Rotaclone; Meridian Bioscience Inc.).

|| By enzyme immunoassay (Premier Adenoclone; Meridian Bioscience Inc.).

- By rapid immunoassay (ImmocoCardSTAT! Crypto/ Giardia; Meridian Bioscience Inc.).

\# By direct smear.

65 unique patients. One patient had two positive specimens 3 weeks apart (both GI.6) and another patient had three specimens 3 weeks and 3 months apart (GII.7, GII.4 and GII.7).

NoV showed a distinct seasonal distribution with 46 $(67 \cdot 6 \%)$ of the cases presenting between October and January (Fig. 1). NoV cases appeared later in the year compared to the peak season for Shigella (JulySeptember, $P<0 \cdot 0001$ ) or Salmonella (July-October, $P=0.003$ ) and earlier than RV (March-April, $P=$ $0 \cdot 02$ ); adenovirus, $C$. difficile and pathogen-negative cases had no clear seasonality or a bi-modal pattern. Similarly, there was a distinct age distribution for NoV with $50(73 \cdot 5 \%)$ of the cases presenting in children aged 6-48 months (Fig. 2). Children aged $<4$ years had NoV detected about four times more frequently than older children $(11.6 \%$ vs $2.9 \%, P<$ $0 \cdot 0001)$. The age of children affected by NoV [median
$1 \cdot 8$, interquartile range (IQR) $0 \cdot 9-3 \cdot 1$ years] was significantly younger than those affected by $C$. difficile (6.97, IQR 1.6-15.0 years, $P<0 \cdot 0001)$, Shigella $(5 \cdot 0$, IQR 3.3-6.8 years, $P<0 \cdot 0001)$ or pathogen-negative (3.61, IQR $0 \cdot 6-12 \cdot 8$ years, $P=0 \cdot 008)$ diarrhoea.

Of the $68 \mathrm{NoV}$-positive patients from whom clinical data was available, diarrhoea was present in $90.7 \%$ (49/54), vomiting in $86 \cdot 8 \%(46 / 53)$, fever in $58 \cdot 8 \%$ (30/51), and dehydration in $48 \cdot 7 \%$ (19/39). Twenty-one of the $68(30.9 \%) \mathrm{NoV}$-positive patients were admitted; $52 \cdot 2 \%(12 / 23)$ in those with underlying illness and $25 \cdot 0 \%(5 / 20)$ in those without underlying illness $(P=0 \cdot 13)$. Faecal occult blood was investigated in 36 patients and six $(16 \cdot 7 \%)$ were positive, with two having an alternative pathogen detected (one Salmonella sp. and one Shigella sonnei).

\section{DISCUSSION}

The main objectives of our study were to determine the prevalence of NoV infection in children attending for gastroenteritis at our hospital, as well as its seasonal and age distribution.

We found an overall NoV prevalence of 7.6\% (68/ 892). Few other studies have looked into endemic NoV infection in paediatric US populations. Similar to ours, all these studies were hospital-based, spanned at least 1 year and evaluated specimens submitted for stool pathogens using RT-PCR. One study [10] restricted to children aged 15 days to 5 years found $\mathrm{NoV}$ in $7 \cdot 1 \%(131 / 1840)$ of specimens; and two other studies evaluating all paediatric age groups found $\mathrm{NoV}$ in $11 \%(352 \mathrm{of} / 3222)$ and $10 \%(98 / 941)$ of specimens $[15,16]$. In contrast, two other studies reported a somewhat higher NoV prevalence. One study [17] was restricted to children aged $<5$ years and found $\mathrm{NoV}$ in $21 \%$ (278/1295), and the other study [18] included all paediatric age groups and found $17 \%(30 / 172)$ of the specimens positive for NoV. The difference in NoV prevalence in the studies is likely a reflection of the differences in the populations studied, the selection criteria used (for the specimens and subjects to be tested) and the local and year-to-year variation in NoV activity.

While hospital-based surveillance may not be the best methodology to study the full spectrum of NoV illness, it has proven most convenient for specimen collection and testing. Community-based studies are not practical at the present time, mainly because of lack of a simple and accurate test to detect NoV; currently, conventional RT-PCR or real-time RT-PCR 




Fig. 1. Number of specimens tested for norovirus (light columns) and percent positivity rate (dark columns) by month, 2011-2012.

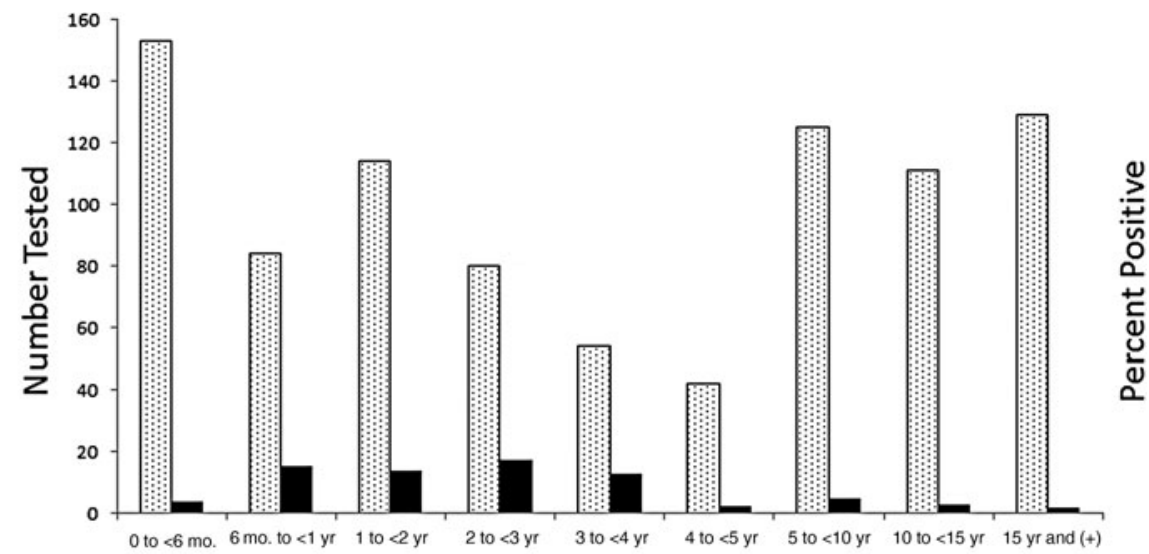

\begin{tabular}{|l|c|c|c|c|c|c|c|c|c|}
\hline No. tested & 153 & 84 & 114 & 80 & 54 & 42 & 125 & 111 & 129 \\
\hline Percent positive & 3.9 & 15.5 & 14.0 & 17.5 & 13.0 & 2.4 & 4.8 & 2.7 & 1.6 \\
\hline
\end{tabular}

Fig. 2. Number of specimens tested for norovirus (light columns) and percent positivity rate (dark columns) by age group.

are the most accurate tests but they are rather laborious [19]. Community-based, active surveillance, population studies of NoV have been undertaken in developing countries. For example, in Peru 220 children were followed from birth to 2 years and NoV was found in $23 \%$ of 1495 diarrhoea specimens, resulting in $38 \%$ and $71 \%$ of children with at least one episode of NoV-associated diarrhoea by age 1 and 2 years, respectively [20]. However, these results may not be applicable to the United States.

In our study $\mathrm{NoV}$ was the most common virus detected, surpassing RV (68/892 vs. 16/534).
However, it should be noted, that 2011-2012 was a particularly low year for RV activity, and the relative proportions may vary from year to year. Still, the finding of $\mathrm{NoV}$ preponderance over $\mathrm{RV}$ has also been described by others [15-17] and is a direct consequence of RV vaccination. Yet, even though NoV has become one of the main causes of diarrhoea in children in our area, it still does not have the levels of morbidity and severity that RV used to have. As an example, before the introduction of $\mathrm{RV}$ vaccines in our hospital we used to see about $200 \mathrm{RV}$-positive stool specimens every year and about $57 \%$ of these 
required hospitalization [3]; whereas in this study there were $68 \mathrm{NoV}$-positive patients and 21 (31\%) were hospitalized. It also should be noted that percent-wise C. difficile was the most prevalent pathogen (19.9\%) surpassing NoV $(7 \cdot 6 \%)$. This finding may reflect the result of selective testing and the more widespread use of nuclear amplification assays, which tend to overestimate the importance of $C$. difficile as a pathogen [21].

NoV infections showed a clear seasonal distribution (Fig. 1); the average positivity rate was $14 \cdot 6 \%(46 / 314)$ for October-January while it was $3 \cdot 3 \%(22 / 661)$ for the rest of the year; this seasonality has also been described by others [17, 18]. NoV was more common in specimens obtained from children aged $<4$ years $(56 / 485,11.6 \%)$ and especially in those aged 6 months to $<4$ years $(50 / 332,15 \cdot 1 \%)$ (Fig. 2). The age distribution suggests partial protection from maternal antibodies in the first 6 months of life that subsequently wanes and is followed by natural infection and development of own immunity. Yet the presence of NoV infections in children aged $<6$ months or $>4$ years, without much of a significant change in the detection rates with increasing age would indicate incomplete protection and suggest that a potential NoV vaccine would need to confer long-term protection to have an impact on the epidemiology of NoV disease. In fact, initial studies based on human challenge experiments suggested immunity lasted only between 6 months and 2 years [22, 23], while a more recent study, based on clinical transmission models has suggested a somewhat longer but still limited duration of immunity of $4 \cdot 1-8 \cdot 7$ years [24].

A number of limitations of the present study must be recognized. First, this was a hospital-based surveillance, and as such it captures medically attended gastroenteritis but not cases of lesser severity attended in the community, and the lack of a denominator (catchment population) does not allow for population incidence estimates. Second, our data applies to paediatric patients only and to sporadic (not outbreak) diarrhoea (while we cannot be entirely certain, to our knowledge none of our cases was epidemiologically linked to an outbreak). Third, the study includes children seen at a single centre and for a single 12-month period; so the applicability of the findings for other locales or other years cannot be determined. Fourth, while the collection of stool specimens was prospective, the collection of clinical data was retrospective and not standardized; so, we had no control over data documentation or procedures, resulting in some variables being missing or imprecise (and hence, not used for analysis). Fifth, the study used specimens originally obtained for testing of other pathogens, so a selection bias (based on what clinicians thought likely aetiologies) may have been introduced. Indeed, not all specimens were tested for all pathogens making comparisons difficult; for example all specimens were tested for NoV (making its number higher), while others (e.g. C. difficile toxin) were tested more selectively based on clinical presumption (and possibly making its rates higher).

Despite these limitations, we believe our data shows that NoV has become one of the main causes of community-acquired gastroenteritis in the United States and the number 1 cause of viral diarrhoea, especially in young children. These findings may also be applicable to other countries with widespread immunization against $R V$.

\section{ACKNOWLEDGMENTS}

We thank Dr Lorna Seybolt and Ms Lauren Hayes for assistance in data collection; we also thank the laboratory personnel at Children's Hospital New Orleans for their assistance in the collection of stool specimens. This research was supported by an Intramural Grant from the Department of Pediatrics, Louisiana State University Health Sciences Center at New Orleans, Louisiana, USA.

\section{DECLARATION OF INTEREST}

None.

\section{REFERENCES}

1. United Nations Children's Fund. Levels and trends in child mortality. Report 2014 (http://www.data.unicef. org/corecode/uploads/document6/uploaded_pdfs/coreco de/Child_Mortality_Report_2014_195.pdf). Accessed 3 August 2015.

2. Glass RI, et al. Rotavirus vaccines: targeting the developing world. Journal of Infectious Diseases 2005; 192: S160-S166.

3. Bégué RE, Perrin K. Reduction in gastroenteritis with the use of pentavalent rotavirus vaccine in a primary practice. Pediatrics 2010; 126: e40-e45.

4. Leshem E, et al. Rotavirus vaccines and health care utilization for diarrhea in the United States (2007-2011). Pediatrics 2014; 134: 15-23.

5. Karafillakis E, Hassounah S, Atchison C. Effectiveness and impact of rotavirus vaccines in Europe, 20062014. Vaccine 2015; 33: 2097-2107. 
6. Kapikian AZ, et al. Visualization by immune electron microscopy of a $27 \mathrm{~nm}$ particle associated with acute infectious nonbacterial gastroenteritis. Journal of Virology 1972; 10: 1075-1081.

7. Glass RI, et al. The epidemiology of enteric caliciviruses from humans: a reassessment using new diagnostics. Journal of Infectious Diseases 2000; 181 (Suppl. 2): S254-S261.

8. Widdowson MA, Monroe SS, Glass RI. Are noroviruses emerging? Emerging Infectious Diseases 2005; 11: 735737.

9. Patel MM, et al. Systematic literature review of role of noroviruses in sporadic gastroenteritis. Emerging Infectious Diseases 2008; 14: 1224-1231.

10. Zintz C, et al. Prevalence and genetic characterization of caliciviruses among children hospitalized for acute gastroenteritis in the United States. Infection, Genetics and Evolution 2005; 5: 281-290.

11. Ahmed SM, et al. Global prevalence of norovirus in cases of gastroenteritis: a systematic review and meta-analysis. Lancet Infectious Diseases 2014; 14: 725-730.

12. Gastañaduy PA, et al. Burden of norovirus gastroenteritis in the ambulatory setting-United States, 20012009. Journal of Infectious Diseases 2013; 207: 10581065.

13. Kageyama $\mathbf{T}$, et al. Broadly reactive and highly sensitive assay for Norwalk-like viruses based on real-time quantitative reverse transcription PCR. Journal of Clinical Microbiology 2003; 41: 1548-1557.

14. Kojima $\mathbf{S}$, et al. Genogroup-specific PCR primers for detection of Norwalk-like viruses. Journal of Virological Methods 2002; 100: 107-114.
15. Koo HL, et al. Noroviruses: the most common pediatric viral enteric pathogen at a large university hospital after introduction of rotavirus vaccination. Journal of the Pediatric Infectious Diseases Society 2013; 2: 57-60.

16. Osborne CM, et al. Viral gastroenteritis in children in Colorado, 2006-2009. Journal of Medical Virology 2015; 87: 931-939.

17. Payne DC, et al. Norovirus and medically attended gastroenteritis in US children. New England Journal of Medicine 2013; 368: 1121-1130.

18. Chamberland RR, et al. Prevalence and seasonal distribution of norovirus detection in stools submitted from pediatric patients for enteric pathogen testing. Journal of the Pediatric Infectious Diseases Society 2015; 4: 264-266.

19. Yen C, Hall AJ. Challenges to estimating norovirus disease burden. Journal of the Pediatric Infectious Diseases Society 2013; 2: 61-62.

20. Saito M, et al. Multiple norovirus infections in a birth cohort in a Peruvian periurban community. Clinical Infectious Diseases 2014; 58: 483-491.

21. Polage $\mathbf{C}$, et al. Overdiagnosis of Clostridium difficile infection in the molecular test era. JAMA Internal Medicine 2015; 175: 1792-1801.

22. Parrino TA, et al. Clinical immunity in acute gastroenteritis caused by Norwalk agent. New England Journal of Medicine 1977; 297: 86-89.

23. Johnson PC, et al. Multiple-challenge study of host susceptibility to Norwalk gastroenteritis in US adults. Journal of Infectious Diseases 1990; 161: 18-21.

24. Simmons K, et al. Duration of immunity to norovirus gastroenteritis. Emerging Infectious Diseases 2013; 19: $1260-1267$. 